\title{
Angst vor dem Kannibalen: Zum Motiv der Anthropophagie in der Universalge- schichte des Nikolaos von Damaskus (FGrHist 90 F 1-102)
}

\author{
Tino Shahin
}

\section{Inhaltsangabe}

In mehreren Fragmenten der Universalgeschichte des Nikolaos von Damaskus (64-4 v. Chr.) werden Fälle von Anthropophagie thematisiert. Diese Überlieferungen gehen zwar auf ältere Quellen zurück (schließlich hat der Geschichtsschreiber sein Werk weitgehend kompiliert), allerdings wählte Nikolaos seine Vorlagen bewusst aus und setzte individuelle Akzente, sodass die Betrachtung von Erzählmotiven zu einer Erschließung der Universalgeschichte beitragen kann. Die Belege für Anthropophagie bei Nikolaos werden hier erstmals zusammengestellt und untersucht. Im Zentrum der Analyse steht die Frage nach dem diskursiven Umgang des Historikers mit dem Phänomen sowie nach der Funktion des Narrativs in seinem Werk.

\section{Einführung}

Obwohl von der ursprünglich 144 Bücher umfassenden Universalgeschichte ${ }^{1}$ des Nikolaos von Damaskus (64-4 v. Chr.) lediglich 102 Fragmente überliefert sind (so zumindest nach der immer noch maßgeblichen Sammlung FGrHist von Felix Jacoby), fällt auf, dass es in den erhaltenen Textauszügen eine Reihe wiederkehrender Motive gibt. Mehrfach finden sich etwa Beschreibungen und Andeutungen von Kannibalismus, also des Verzehrs von Artgenossen, oder genauer: von Anthropophagie, also des Verspeisens von Menschenfleisch durch Menschen. Vorstellungen über das Phänomen fanden zeitlich wie geographisch eine universale Verbreitung. ${ }^{2}$ Schon in den frühesten griechischen Quellen wurde es den Grundwerten der griechischen Kultur entgegengestellt und als ,entsetzlich“ bewertet. ${ }^{3}$ Neben Ablehnung äußert sich in vielen Überlieferungen aber auch eine gewisse Faszination für Anthropophagie - zumindest kann dieser Eindruck in Anbetracht der Zahl der Quellen und ihrer Gestaltung entstehen.

Die Erwähnungen bei Nikolaos sind auf seine Vorlagen zurückzuführen; in seiner Zeit gab es wohl kaum Fälle von Kannibalismus, und der Vertraute des Herodes konnte den großen Umfang seines Geschichtswerks nur dadurch erreichen, dass er

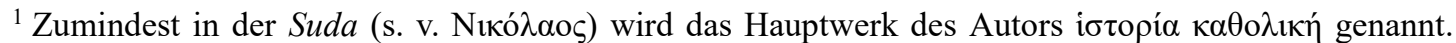
Aufgrund der zeitlichen und geographischen Breite der Darstellung erscheint die Bezeichnung „Universalgeschichte“ sinnvoll.

${ }^{2} \mathrm{Zu}$ Anthropophagie in der Antike vgl. den Sammelband von PöHL/FINK (2015) und den älteren Lexikonartikel von KURFEß (1950). Großes Aufsehen erregte die Arbeit von ARENS (1979), in der er die Historizität aller Überlieferungen zu Anthropophagie in Frage stellte. Zum Motiv in der neuzeitlichen Literatur vgl. HEESEN (2008) und KECK ET AL. (1999). Zum Thema „Kannibalismus und europäische Kultur"vgl. den Sammelband von RÖCKELEIN (1996).

${ }^{3}$ Die Taten des Kyklopen Polyphem, der Gefährten des Odysseus verspeist, gelten bei Homer als $\sigma \chi \varepsilon \dot{\varepsilon} \tau \lambda 1 \alpha$ und werden damit erklärt, dass er sich nicht um Gastfreundschaft oder die Götter kümmere (Od. 9,273-295). Nach Hesiod hatte der Göttervater Kronos zum Leid der schwangeren Rheia vor, seine eigenen Kinder $\mathrm{zu}$ verschlingen (theog. 466-467). Herodot erwähnt etwa das Volk der „Androphagen“, das weder Recht noch Gesetz kenne $(4,18 ; 4,106)$.
} 
über weite Strecken kompilatorisch gearbeitet hat. Jeder Übernahme einer früheren Darstellung liegt jedoch eine Entscheidung des Autors zugrunde, der seine Quellen bewusst auswählte und individuelle Akzente setzte. Obwohl das Werk des Nikolaos seit einigen Jahren durch eine französische Übersetzung aller Fragmente einem größeren Forscherkreis zugänglich ist, hält sich die Zahl der bisher erschienenen Untersuchungen zu ihm in Grenzen. ${ }^{4}$ Unter dem Aspekt Anthropophagie wurden seine Überlieferungen bisher noch nicht betrachtet. In diesem Beitrag werden die entsprechenden Fragmente herangezogen, um über die Frage nach dem diskursiven Umgang des Historikers mit diesem Phänomen einen Zugang zur größtenteils verlorenen Universalgeschichte zu eröffnen. Für die Betrachtung wird weniger der Vergleich der Fragmente mit den Parallelüberlieferungen als die Funktion des Erzählmotivs innerhalb des Geschichtswerks des Autors eine Rolle spielen.

\section{Das Motiv der Anthropophagie in der Universalgeschichte}

Ein erster Beleg für Anthropophagie findet sich in Fragment 22 des Nikolaos - einem Exzerpt, das im 10. Jahrhundert unter dem byzantinischen Kaiser Konstantinos VII. Porphyrogennetos zum vierten beziehungsweise fünften Buch der Universalgeschichte angefertigt wurde. ${ }^{5}$ Das Geschehen lässt sich folgendermaßen zusammenfassen: Der Lyderkönig Kamblitas soll so ungemein gefräßig gewesen sein, dass ihm sogar das Verlangen kam, seine eigene Frau aufzuessen. Er selbst meinte, unter dem Einfluss eines Gifts gestanden zu haben, und ging nach Bekanntwerden der Tat mit einem Schwert auf den belebten Marktplatz, wo er sich nach einem kurzen Gebet zu Zeus umbrachte. Die einen verspotteten ihn darauf als Verfressenen, die anderen bemitleideten ihn aber als Wahnsinnigen, der (möglicherweise von Iardanos) vergiftet worden war.

Die Darstellung ist zeitlich nicht näher einzuordnen, entstammt aber einem Teil des Werks über die lydische Geschichte. ${ }^{6}$ Der Herrscher, um den es geht, kommt unter dem Namen Kambles auch bei Xanthos vor, der die Geschichte ähnlich erzählt wie Nikolaos und vermutlich dessen Vorlage war. $^{7}$ Nikolaos legt sich nicht auf eine Erklärung für den Kannibalismus fest, sondern gibt nur die geäußerten Ansichten

\footnotetext{
${ }^{4}$ PARMEntier/BArone (2011). Die letzte Monographie zu Nikolaos stammt von WaChOlder (1962). Einige Aufsätze gaben in den letzten Jahrzehnten einen Überblick über die Fragmente der Universalgeschichte, vgl. ALONSO-NúÑEZ (1995) und TOHER (1989). Weiterhin erschien eine Reihe von Untersuchungen zu einzelnen Aspekten des Werks, vgl. etwa zur lydischen Geschichte PARMENTIER (1995) oder zu den Abhängigkeiten des Nikolaos von Ktesias LENFANT (2000) sowie LENFANT (2012).

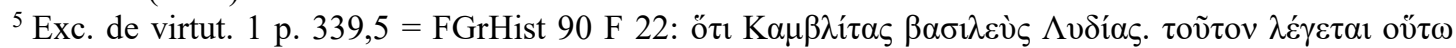

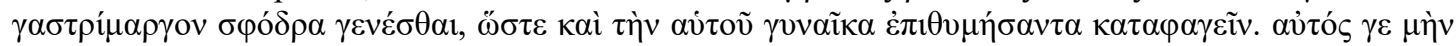

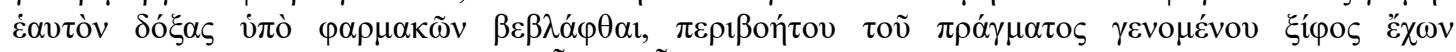

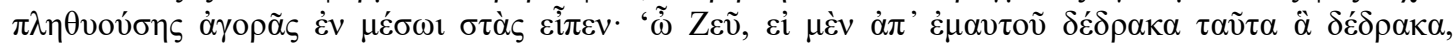

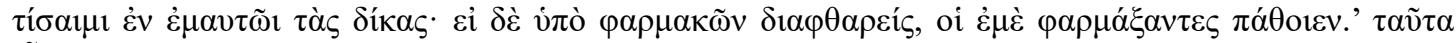

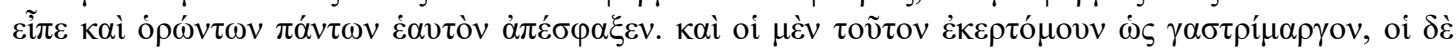

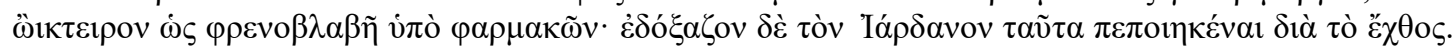
Eine erste deutsche Übersetzung der Fragmente zur Universalgeschichte des Nikolaos erscheint in Kürze beim Hiersemann Verlag: T. Shahin, Nikolaos von Damaskus (Fragmente der Historiker), Stuttgart 2018.

${ }^{6}$ Berücksichtigt man die Reihenfolge, in der die Fragmente des Nikolaos in den byzantinischen Exzerpten überliefert sind, ist am wahrscheinlichsten, dass Kamblitas ein Nachfahre des Atys war (F 15); vgl. PARMENTIER/BARONE (2011) 60; SCHUBERT (1884) 5.

${ }^{7}$ FGrHist 765 F 18 = Athen. 10,8 (415c-d). Vgl. zu dem Lyderkönig auch Ael. VH 1,27 sowie Eustath. Hom. 1630,15 (Rhaps. 9,356,17).
} 
durch die Worte oi $\mu \varepsilon ́ v$ [...] oi $\delta \varepsilon ́$ wieder. Gemäß der ersten Meinung handelte sich um einen Fall der Polyphagie. Dem König wurde demnach vorgeworfen, dass er vorsätzlich eine Schandtat begangen habe. Der zweiten Meinung zufolge, die Kamblitas selbst vertrat, erfolgte die Tat infolge einer Vergiftung und der daraus resultierenden geistigen Beeinträchtigung - also ohne Absicht. Im Zentrum der Handlung steht aber die Ungewissheit, was Kamblitas zum Verspeisen seiner Frau verleitet hat. Die Erwähnung des Iardanos, dessen Tochter Omphale ein Verhältnis mit Herakles hatte, weist eine Verbindung der Geschichte mit dem griechischen Mythos auf (zur Deutung s.u.).

„Unbeabsichtigte“ Anthropophagie wird auch in Fragment 38 des Nikolaos thematisiert. ${ }^{8}$ Die Überlieferung stammt ebenfalls aus byzantinischer Zeit und gibt einen Teil aus dem fünften Buch der Universalgeschichte wieder. Zusammengefasst lässt sich dem Fragment entnehmen, dass Lykaon, der König von Arkadien, an den Vorschriften seines Vaters Pelasgos in Hinsicht auf die Gerechtigkeit festhielt. Da Lykaon auch seine Untertanen vom Unrecht abbringen wollte, behauptete er, dass Zeus ihn als Gastfreund besuche, um die Gerechten von den Ungerechten zu unterscheiden. Weil einige der 50 Söhne des Königs, die er von verschiedenen Frauen hatte, wissen wollten, ob wirklich ein Gott zu ihnen komme, töteten sie einen Knaben und vermengten sein Fleisch mit dem eines Opfertieres. Dafür richtete die Gottheit sie mit Blitzen zugrunde.

In diesem Fragment, das aus einer Darstellung des Nikolaos über das frühe Arkadien stammt, wird der Mythos von Lykaon aufgegriffen, allerdings weicht er in seiner Erzählung von anderen antiken Autoren ab, sodass auch seine Vorlage nicht zu bestimmen ist. ${ }^{9}$ Im Gegensatz zu den Parallelüberlieferungen bleibt Lykaon in dieser Variante unschuldig, dafür werden ihm einige seiner Nachkommen gegenübergestellt, welche die väterlichen Vorschriften missachten. Der Zweifel an der Gegenwart des Gottes verleitet einige Söhne zu dem Versuch, Zeus auf die Probe zu stellen, indem sie beim Opfermahl Menschenfleisch als Speise vorlegen. Während Kamblitas in Fragment 22 selbst einen Fall von Kannibalismus begeht, wollen die Lykaon-Söhne hier also den Gastfreund zum Anthropophagen machen. Erneut hat ein Frevel den Tod zur Folge, wobei es dieses Mal eine Gottheit ist, welche die Sanktion vollzieht. Das Motiv, dass Göttern ein Kind als Mahl vorgesetzt wird, ist aus dem griechischen Mythos bekannt: Nach einer Tradition, die Pindar wiedergibt, habe Tantalos ihnen seinen Sohn Pelops als Speise vorgelegt. ${ }^{10}$

Gegenüber den angeführten Fragmenten, die aus den Büchern des Nikolaos zur Frühgeschichte stammen, thematisiert Fragment 93, welches bei Flavius Josephus erhalten

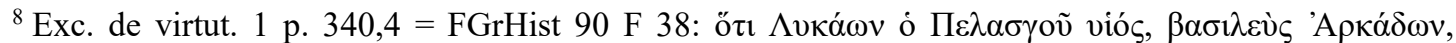

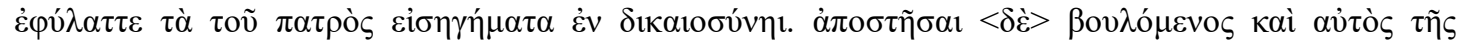

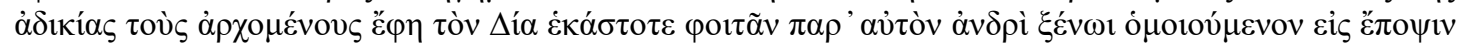

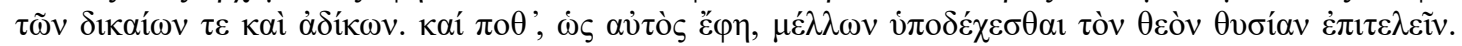

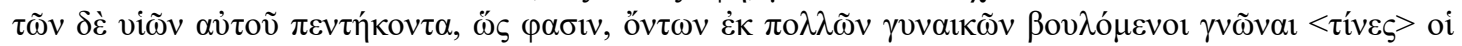

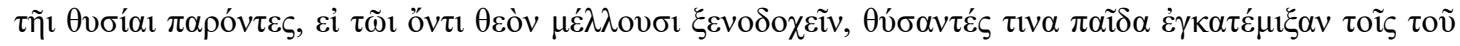

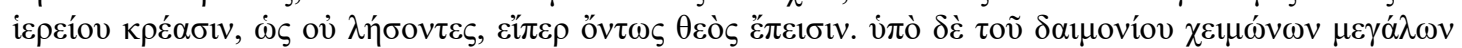

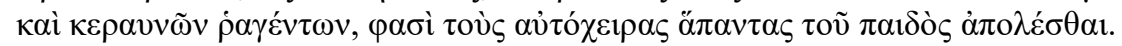

${ }^{9}$ Lykaon wird in mehreren Quellen in Verbindung mit einem Menschenopfer gebracht. Außerdem soll er sich in einen Wolf verwandelt haben, vgl. Paus. 8,2,3-4; Ov. met. 1,237; Hyg. fab. 177; Apollod. bibl. 3,99 (3,8,1); Nonn. 18,20-24. Zum Lykaon-Mythos vgl. Burkert (1972) 98-108.

${ }^{10}$ Pind. O. 1,37-66.
} 
ist, ein Ereignis aus der Diadochenzeit. ${ }^{11}$ Josephus gibt hier nicht an, welches Buch der Universalgeschichte ihm als Vorlage diente. Das Fragment muss aber auf einen späten Teil des Werks zurückgehen, denn die im Textauszug thematisierte Auseinandersetzung ereignete sich nur wenige Jahrzehnte vor der Lebenszeit des Nikolaos, der in seinem Werk weitgehend chronologisch gearbeitet hat: ${ }^{12}$

Dem Fragment ist zu entnehmen, dass sich Ptolemaios IX. am Abend nach dem Sieg gegen Alexander Iannaios in Judäa niederließ. Er befahl seinen Soldaten, alle Frauen und Kinder in den besetzten Dörfern zu töten, zu zerstückeln und die Körperteile als Opfer in Kessel mit siedendem Wasser zu werfen. Ptolemaios ordnete dies an, damit die Feinde, die fliehen konnten, sie für Menschenfresser hielten und dadurch in Schrecken gerieten. Am Ende des Fragments verweist Josephus auch auf eine Darstellung von Strabon, allerdings ist diese wie diejenige des Nikolaos verloren. ${ }^{13}$

Die Handlung ist relativ genau in die Zeit zwischen 103 und $101 \mathrm{v}$. Chr. einzuordnen, als der hasmonäische König Alexander Iannaios sein Reich an der Mittelmeerküste und im Ostjordanland ausdehnte. Die Bürger der Stadt Ptolemais baten Ptolemaios IX. um Hilfe, der infolge eines Machtkampfes aus Ägypten nach Zypern geflohen und nach Syrien übergesetzt war, um gegen den Hasmonäer vorzugehen. Ptolemaios wandte dabei übermäßige Gewalt an, indem er eine Vielzahl von Frauen und Kindern

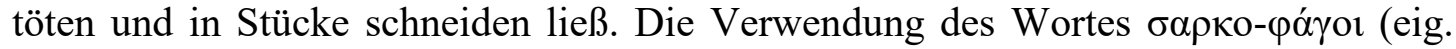
„Fleischfresser") in $\S 346$ anstelle von $\alpha \dot{\alpha} \theta \rho \omega \pi \mathrm{o}-\varphi \alpha ́ \gamma o r$ soll vielleicht deutlich machen, dass er seine Opfer unmenschlich behandelt hat. ${ }^{14}$

\section{Analyse: Zur Angst vor dem Kannibalen}

In den drei angeführten Fragmenten der Universalgeschichte ist das Motiv der Anthropophagie zentral für die jeweilige Handlung: sei es, dass ein Fall von Kannibalismus erfolgt (wie beim Lyderkönig Kamblitas) oder ein solcher droht (wie bei den Söhnen des Lykaon) beziehungsweise vorgetäuscht wird (wie im Falle des Ptolemaios IX.). Das Vergehen des Kamblitas (F 22) erscheint besonders verwerflich, weil es sich bei dem Opfer um seine eigene Frau handelt. ${ }^{15}$ Für die negative Bewertung der Tat - der Herrscher sieht keinen anderen Ausweg, als sich selbst zu töten - spielt ihre Wehrlosigkeit und Unschuld eine Rolle. Das Mitgefühl, das so evoziert wird, lädt die Handlung emotional auf. ${ }^{16}$ Unter diesem Aspekt zeigt sich neben der Erwähnung des

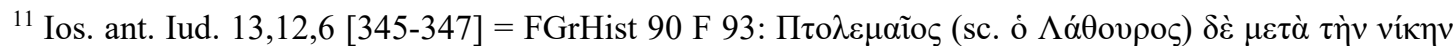

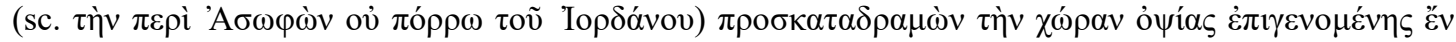

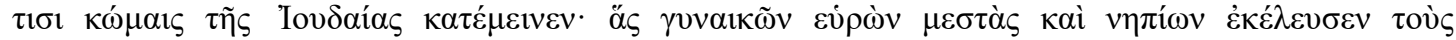

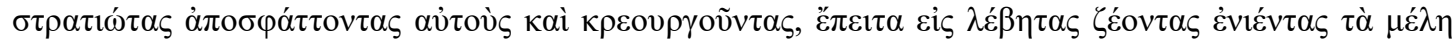

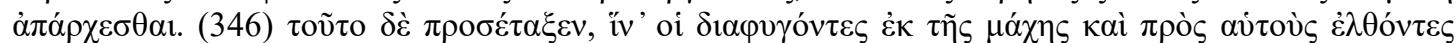

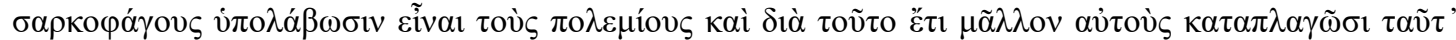

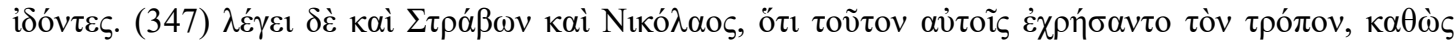

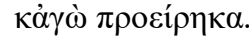

${ }^{12} \mathrm{Zu}$ seiner Arbeitsweise nach geographischen und chronologischen Kriterien vgl. ALONSO-NúÑEZ (1995) 12-13.

${ }^{13}$ FGrHist 91 F 12. Im Jüdischen Krieg des Josephus existiert eine weitere kurze Erwähnung des Konflikts, vgl. Ios. bell. Iud. 1,4,2 (86-87).

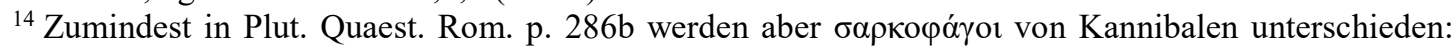

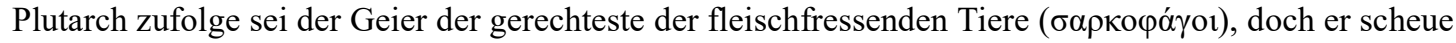
alles, was gleichen Geschlechtes sei.

${ }^{15}$ Zum Motiv der Anthropophagie unter Angehörigen vgl. BORSAY (1965) 80-81.

${ }^{16}$ Nach Zimmermann stehe das Handeln des Kamblitas auch mit seiner Reue und der Trauer um seine
} 
Iardanos eine weitere Verbindung des Kamblitas zu Herakles, der ebenfalls in der eigenen Familie mordet und anschließend Reue zeigt. Wehrlos und unschuldig erscheinen bei Nikolaos aber auch die Opfer in den anderen beiden Überlieferungen, schließlich geht es um die Tötung von Frauen und Kindern. Anders als im KronosMythos sind es in den Fragmenten der Universalgeschichte aber nicht die eigenen Söhne, die verschlungen werden. ${ }^{17}$

Neben der Rolle der Opfer besteht eine weitere Parallele zwischen den Textauszügen über den lydischen Herrscher und die Söhne des Lykaon darin, dass von der Gefahr der unwissentlichen Anthropophagie ausgegangen wird. Dieses Motiv ist aus dem griechischen Mythos bekannt und wurde im sogenannten „Mahl des Thyestes“ verarbeitet: Atreus hatte dabei seinem Bruder dessen Söhne als Speise vorgelegt. ${ }^{18}$ Unter den Geschichtsschreibern berichtete schon Herodot, dass der Mederkönig Astyages seinen Untertanen Harpagos unwissentlich den eigenen Sohn essen ließ. Harpagos war nämlich nicht dem Befehl nachgekommen, den jungen Kyros, dem die Machtübernahme prophezeit worden war, zu beseitigen. ${ }^{19}$ Bei Herodot findet sich auch eine Parallele zu dem Motiv in Fragment 93, dem Töten von Kindern des Gegners. ${ }^{20}$ Weil es in der Erzählung von Herodot aber keinen Fall des Kannibalismus gibt, ist eine Verbindung zur Überlieferung des Nikolaos unwahrscheinlich.

Kamblitas, dem möglicherweise eine Bestrafung vonseiten der Lyder droht, ${ }^{21}$ entschließt sich, seinem Leben selbst ein Ende zu bereiten und sich als Opfer eines Giftanschlags darzustellen. Dass er sich mitten auf der Agora umbringt, steht in Zusammenhang mit seinem ,sozialen Tod“, der durch den Verlust seiner Ehre infolge des Bekanntwerdens der Tat bereits erfolgt ist. Im Falle der Lykaon-Söhne wird die Wichtigkeit ihrer Bestrafung durch den Eingriff der Gottheit hervorgehoben. Diskursiv weisen beide Geschichten auf die Folgen schwerwiegender Vergehen hin: Nach Nikolaos ist ein Verhalten, das den Grundwerten der Gemeinschaft entgegensteht, mit der Exklusion aus der sozialen Gruppe zu sanktionieren. Der Autor fordert also die Beseitigung des Täters, weil eine Reintegration aussichtslos erscheint. In Hinsicht auf die Struktur der Universalgeschichte sollten die Darstellungen Umbrüche in Lydien und Arkadien erklären, schließlich werden die Fälle von Anthropophagie in Verbindung mit den Machtwechseln gebracht. Der Kannibalismus geht mit einer historischen Zäsur einher, in deren Folge vielleicht frühere (bessere) Verhältnisse unter einer neuen Herrschaft wiederhergestellt werden: Kamblitas nimmt sich selbst das Leben über einen Sohn wird nicht berichtet - und im Falle der Lykaoniden ist anzunehmen, dass jene Söhne die Herrschaft übernahmen, die sich nicht am Frevel beteiligt hatten, denn es ist explizit erwähnt, dass ,einige“ der 50 den Gott prüfen wollten.

\footnotetext{
geliebte Frau in Verbindung, vgl. ZIMMERMANN (2013) 138. Die Unterschiede zwischen der Darstellung des Nikolaos und der Vorlage bei Xanthos erklärt Zimmermann mit einem sinkenden Vergnügen an Gewaltbildern zwischen dem 5. und 1. Jh. v. Chr. Die These, die im Allgemeinen zutreffen mag, ist - allein auf Nikolaos bezogen - aufgrund des fragmentarischen Charakters der Überlieferungen von Nikolaos und Xanthos nicht $\mathrm{zu}$ stützen, zumal es bei Nikolaos andere Darstellungen exzessiver Gewalt gibt (s.u.).

${ }^{17}$ Hes. theog. 466-468.

${ }^{18}$ Aischyl. Ag. 1096f.

${ }^{19}$ Hdt. 1,118-119. Zu Thyestes und Harpagos vgl. BURKERT (1972) 119-125.

${ }^{20}$ Nach Hdt. 3,11 töteten ägyptische Söldner vor den Augen des Verräters Phanes dessen Kinder; vgl. PARMENTIER/BARONE (2011) 169.

${ }^{21}$ So auch ZIMMERMANN (2009) 164.
} 
Fragment 93 unterscheidet sich in einem wichtigen Aspekt von den anderen Überlieferungen. Die Darstellung weist nämlich keine Verbindung zum Mythos auf, sondern dient der Beschreibung eines geschichtlichen Ereignisses. Mit Nikolaos' zeitlicher und geographischer Nähe zu dem Vorfall hängt zusammen, dass die Anthropophagie, die in der hellenistischen Welt eigentlich keinen Platz hat, von Ptolemaios nur vorgetäuscht wird. Durch die maßlose Anwendung von Gewalt erscheint die Art seiner Kriegsführung dennoch verwerflich, und die Parallelüberlieferung bei Strabon, die Josephus erwähnt, schien dies ebenfalls nahegelegt zu haben. Offenbar haben die antiken Autoren ein einheitliches Bild des Ptolemäers vermittelt. In Bezug auf Nikolaos ist die Kritik an der militärischen Intervention damit zu erklären, dass er als Vertrauter des Herodes eine enge Verbindung zu Judäa hatte. Strabon könnte wiederum von der Darstellung des Nikolaos beeinflusst worden sein, immerhin ist an anderer Stelle bezeugt, dass er die Universalgeschichte seines Zeitgenossen als Quelle verwendet hat. ${ }^{22}$

Den angeführten Darstellungen des Nikolaos ist gemeinsam, dass sie einen Zusammenhang zwischen Anthropophagie und Angst erkennen lassen: Das Verspeisen von Menschenfleisch war der hellenistischen Welt fremd, und Angst erklärt sich vielfach mit dem Nichtwissen über das Fremde, von dem potenziell Gefahr ausgeht. ${ }^{23}$ Besonders deutlich wird die Verbindung von Anthropophagie und Angst in Fragment 93,

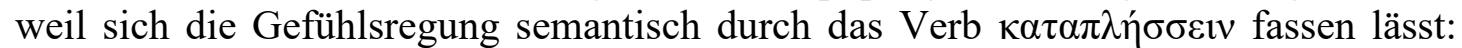
Damit seine Feinde in Schrecken geraten, stellte sich Ptolemaios als Kannibale dar. Der Feldherr will also Angst evozieren, indem er zum Schein Menschenfleisch zubereiten lässt. Der ohnehin fremden Praxis folgt Ptolemaios dabei in einer übersteigerten Form, denn es sind zahlreiche Kinder und Frauen, deren Fleisch gekocht wird.

Zumindest als analytische Kategorie spielt Angst aber auch in den anderen Fragmenten des Nikolaos eine Rolle. Dass sich Kamblitas selbst tötete, könnte mit einer Angst vor Sanktionierung zusammenhängen, denn er hatte eine Schandtat begangen und war zu einem Fremden unter den Lydern geworden. Diese „Entfremdung“ gefährdete die soziale Gruppe, wie am Beispiel seiner Frau demonstriert wird, die dem Täter nahestand und dennoch getötet wurde. Außerdem kommt in der Geschichte ebenso wie in der über die Söhne des Lykaon die Angst zum Ausdruck, unwissentlich Menschenfleisch zu verspeisen. Und schließlich scheint in allen Fragmenten der Aspekt, dass die Todesopfer nicht bestattet werden können, eine Rolle zu spielen. Schon Herodot hat hervorgehoben, wie wichtig den Hellenen eine Bestattung war. ${ }^{24}$ Darstellungen über den drohenden Verstoß gegen diese Sitte könnten beängstigend gewirkt haben.

\footnotetext{
${ }^{22}$ Strabon beruft sich auf einen Bericht des Nikolaos, der im Jahre 20/19 v. Chr. in Antiochia bei Augustus war, als eine indische Gesandtschaft zum Princeps kam, vgl. Strab. 15,1,73 [719C,21$720 \mathrm{C}, 7]=\mathrm{F} 100$ des Nikolaos.

${ }^{23}$ Angst wird hier als Oberbegriff einer Reihe bedrückender Gefühlsregungen verstanden. Eine Verbindung der Emotion zur Anthropophagie lässt sich schon aus dem Kronos-Mythos deuten, denn der Titan beabsichtigt, seinen Sohn Zeus zu verschlingen, und ruft durch die drohende Gefahr das Leid der Rheia hervor, vgl. Hes. theog. 466-468. Zu Emotionen allgemein vgl. CAIRNS/NELIS (2017); SANDERS/JohnCOCK (2016); CHANIOTIS/DuCREY (2012); PlAMPER (2012). Speziell zur Angst vgl. KNEPPE (1996). Zum Aspekt Fremdheit vgl. etwa MÜNKLER (2008); STENGER (1997).

${ }^{24}$ Herodot hatte die Hellenen, die um keinen Preis ihre verstorbenen Väter verspeisen würden, von den Kallatiern und den Issedonen unterschieden, bei denen dies Sitte sei $(3,38 ; 4,26)$.
} 


\section{Auswertung}

Jedem Fragment liegt ein Interesse des Autors zugrunde, der die Schriften des Nikolaos als Quelle verwendet hat. Gleichwohl lässt sich erkennen, dass es mehrere Beschreibungen und Andeutungen von Anthropophagie in der Universalgeschichte des Nikolaos gab. Belege für diese Darstellungsmittel finden sich in den Büchern 4 und 5 sowie in deutlich späteren Teilen des Werks. Fragment 93 ist für diese Feststellung entscheidend, denn bei einem Großteil der Überlieferungen zur Universalgeschichte handelt es sich um sogenannte konstantinische Exzerpte, die den ersten sieben Büchern des Nikolaos zugeordnet sind. Es gibt aber noch weitere Fragmente, die über das Zerschneiden von Körpern berichten und dadurch zumindest Assoziationen mit Anthropophagie wecken: So soll Medea, laut Fragment 54, die Töchter des Pelias dazu verleitet haben, ihren Vater in Stücke zu schneiden und in einen Kessel mit kochendem Wasser hineinzugeben.

Auffällig ist, dass Nikolaos die meisten Darstellungen mit dem Kannibalismus-Motiv auf längst vergangene Zeiten oder nach Asien projiziert. Eine Erklärung dafür ist, dass er die Frühgeschichte seines Werks mit dem Mythos verbunden hat und die Belege für Anthropophagie auf seine Quellen zurückgehen. Die Verortungen im Osten legen nahe, dass das Motiv der Anthropophagie in Zusammenhang mit einer Barbarentopik steht. ${ }^{25}$ Die zeitliche und geographische Distanz, die Nikolaos zu seinen Handlungen konstruiert hat, lässt darauf schließen, dass es ihm um das Aufzeigen von Gegengesellschaften mit zivilisatorischen Defiziten ging, um eine Identifikation mit griechischen Normen zu fördern. Aus einem Fragment seiner Autobiographie geht hervor, dass er von Herodes zum Verfassen der Universalgeschichte angeregt wurde. ${ }^{26}$ Vermutlich verfolgte Nikolaos, der auch als Ratgeber des Königs tätig war, einen didaktischen Anspruch, der eine Vermittlung von Normen implizierte. ${ }^{27}$

In den Fragmenten 22 und 38 lässt sich weiterhin eine Tyrannen-Kritik erkennen, immerhin berichten sie von Übergriffen unter den Königen Kamblitas und Lykaon, die nicht einmal dazu in der Lage waren, den Frieden in ihren eigenen Häusern zu bewahren. ${ }^{28}$ Durch die Zuschreibung von Grausamkeit und Zügellosigkeit wird das Ende ihrer Herrschaft nicht auf narrativer Ebene nur symbolisch vorausgedeutet, sondern vom Standpunkt des Nikolaos rückblickend erklärt und in gewisser Weise sogar als Befreiung dargestellt. In diesem Zusammenhang ist auch der Name des arkadischen Königs zu begreifen, der vom griechischen Wort $\lambda$ v́ко für Wolf abgeleitet ist. Sowohl dem Tier als auch dem Herrscher wurden grausame Charaktereigenschaften angedichtet. Die topische Zahl von 50 Kindern, die Lykaon mit verschiedenen Frauen gezeugt haben soll, ist als Hinweis auf seine Maßlosigkeit zu verstehen. Er selbst wurde mit großem Kinderreichtum verbunden, allerdings sind unter seiner Herrschaft andere Kinder getötet worden. Die Überlieferung zeigt schließlich, dass Anthropophagie-Diskurse eine enge Verbindung zu Darstellungen über Menschenopfer hatten.

\footnotetext{
${ }^{25}$ Vgl. KISTLER (2012) 68. Gronau sieht im Motiv der Anthropophagie ein Zeichen für „Rohheit“, vgl. GRONAU (2015) 76-77.

${ }^{26}$ FGrHist 90 F 135.

${ }^{27}$ Aus den Fragmenten der Autobiographie des Nikolaos (F 131-139) geht hervor, dass er den König in seiner Lebensführung beriet. Das Geschichtswerk ist in diesem Kontext entstanden, vgl. F 135.

${ }^{28}$ Parmentier erkennt in Kamblitas ein Beispiel des Nikolaos für einen unmoralischen König, vgl. PARMENTIER (1991) 235. Zur Verbindung von Anthropophagie und Tyrannei vgl. GRONAU (2015) $77-$ 80.
} 
Es sind zahlreiche Quellen über blutige Speiseopfer erhalten, die kannibalische Konnotationen wecken, da entweder ,ein Tier für einen Menschen, oder ein Mensch für ein Tier den rituellen Opfertod" ${ }^{“ 29}$ findet. Fragment 93 weist insofern eine Rationalisierung des Anthropophagie-Narrativs auf, als Ptolemaios das Verspeisen von Menschen nur vorgibt, um seine Feinde in Schrecken zu versetzen.

Alles in allem ist festzuhalten, dass das Motiv der Anthropophagie im Geschichtswerk des Nikolaos (wie in anderen Quellen auch) der nachträglichen Erklärung vergangener Machtwechsel sowie der Konstruktion von Alterität zur Förderung der Identifikation mit den griechischen Normen diente. Darüber hinaus lässt sich vermuten, dass das Narrativ mit den Spannungshöhepunkten der jeweiligen Darstellungen verknüpft war und die Belege für Kannibalismus intratextuelle Bezüge zwischen verschiedenen Teilen der Universalgeschichte herstellten. Eine Untersuchung anderer Erzählmotive, wie der Elemente der tragischen Historiographie in Fragment 68, könnte weiteren Aufschluss über Gestaltungsprinzipien des Nikolaos geben. Dadurch könnten auch die Voraussetzungen verbessert werden, um sein Werk mit den Schriften anderer hellenistischer Geschichtsschreiber wie Diodor und Dionysios von Halikarnassos zu vergleichen.

\section{Zitierte Literatur}

AlONSO-NúÑEZ (1995) José Miguel Alonso-Núñez, Die Weltgeschichte des Nikolaos von Damaskos, in: SStor 27, 1995, S. 3-15.

ARENS (1979)

BORSAY (1965)

BURKERT (1972)

William Arens, The Man-Eating Myth. Anthropology and Anthropophagy, Oxford/New York 1979.

Laszlo A. Borsay, Lydia, its Land and History, Pittsburgh 1965.

Walter Burkert, Homo Necans. Interpretationen altgriechischer Opferriten und Mythen, Berlin/New York 1972.

Cairns/Nelis (2017) Douglas Cairns/Damien Nelis (Hg.), Emotions in the Classical World, Stuttgart 2017.

Chaniotis/Ducrey (2012) Angelos Chaniotis/Pierre Ducrey (Hg.), Unveiling Emotions. Sources and Methods for the Study of Emotions in the Greek World, Bd. 1-2, Stuttgart 2012-2013.

GRONAU (2015)

Martin Gronau, Primitives Essen? Überlegungen zum kulturanthropologischen Beigeschmack klassischer Menschenfresserei, in: Pöhl/Fink (2015) S. 65-100.

HeEsen (2008) Sabine te Heesen, Der Blick in die kannibalische Welt. Anthropophagie in Daniel Defoes Robinson Crusoe, den Reisebeschreibungen zu James Cooks Weltumsegelun-

\footnotetext{
${ }^{29}$ GronaU (2015) 76. Den Zusammenhang zwischen Anthropophagie und Menschenopfer sah auch KURFEß (1950). Zu Menschenopfern im antiken Griechenland vgl. HuGHES (1991). Zu altgriechischen Opferriten und Mythen vgl. BURKERT (1972).
} 
Hughes (1991)

KeCK ET AL. (1999)

KISTLER (2012)

KNEPPE (1996)

KURFEß (1950)

LENFANT (2000)

LENFANT (2012)

MÜNKLER (2008)

PARMENTIER (1991)

PARMENTIER (1995)

PARMENTIER/BARONE (2011) Édith Parmentier/Francesca Prometea Barone, Nicolas de Damas, Oeuvre historique: Histoires, Recueil de coutumes, Vie d'Auguste, Autobiographie (Fragments), Paris 2011.

PLAMPER (2012)

PÖHL/FINK (2015)

RÖCKELEIN (1996)

gen und bei Marquis de Sade, Freiburg i. Br. / Berlin / Wien 2008.

Dennis D. Hughes, Human sacrifice in ancient Greece, London/New York 1991.

Annette Keck/Inka Kording/Anja Prochaska (Hg.), Verschlungene Grenzen. Anthropophagie in Literatur und Kulturwissenschaften, Tübingen 1999.

Erich Kistler, À la lydienne... mehr als nur eine Mode, in: Linda-Marie Günther (Hg.), Tryphe und Kultritual im archaischen Kleinasien - ex oriente luxuria?, Wiesbaden 2012, S. 59-69.

Alfred Kneppe, Metus temporum. Zur Bedeutung von Angst in Politik und Gesellschaft der römischen Kaiserzeit des 1. und 2. Jhdts. n. Chr., Stuttgart 1994.

Anton Kurfeß, Art. Anthropophagie, in: RAC 1, 1950, Sp. 450 .

Dominique Lenfant, Nicolas de Damas et le corpus des Fragments de Ctésias, in: AncSoc 30, 2000, S. 293-318.

Dominique Lenfant, Ctesias and his Eunuchs: a

Challenge for Modern Historians, in: Histos 6, 2012, S. 257-297.

Herfried Münkler, Barbaren und Dämonen: Die Konstruktion des Fremden in Imperialen Ordnungen, in: Jörg Baberowski/Hartmut Kaeble/Jürgen Schriewer (Hg.), Selbstbilder und Fremdbilder. Repräsentation sozialer Ordnungen im Wandel, Frankfurt a. M./New York 2008, S. 153-189.

Édith Parmentier, Rois et tyrans chez Nicolas de Damas, in: Ktèma 16, 1991, S. 229-244.

Édith Parmentier, La Lydie, Hérodote et Nicolas de Damas, in: Ktèma 20, 1995, S. 85-94.

Jan Plamper, Geschichte und Gefühl. Grundlagen der Emotionsgeschichte, München 2012.

Friedrich Pöhl/Sebastian Fink (Hg.), Kannibalismus, eine anthropologische Konstante?, Wiesbaden 2015.

Hedwig Röckelein (Hg), Kannibalismus und europäische Kultur, Tübingen 1996.

SANDERS/JOHNCOCK (2016) Ed Sanders/Matthew Johncock (Hg.), Emotion and Persuasion in Classical Antiquity, Stuttgart 2016. 
SCHUBERT (1884)

STENGER (1997)

TOHER (1989)

WACHOLDER (1962)

ZIMMERMANN (2009)

ZIMMERMANN (2013)
Rudolf Schubert, Geschichte der Könige von Lydien, Breslau 1884.

Horst Stenger, Deutungsmuster der Fremdheit, in: Herfried Münkler/Bernd Ladwig (Hg.), Furcht und Faszination. Facetten der Fremdheit, Berlin 1997, S. 159-221.

Mark Toher, On the Use of Nicolaus' Historical Fragments, in: ClAnt 8, 1989, S. 159-172.

Ben Zion Wacholder, Nicolaus of Damascus, Berkley / Los Angeles 1962.

Martin Zimmermann, Extreme Formen physischer Gewalt in der antiken Überlieferung, München 2009.

Martin Zimmermann, Die dunkle Seite der Antike, München 2013.

\section{Kontakt zum Autor:}

Tino Shahin

Rheinische Friedrich-Wilhelms-Universität Bonn

Institut für Geschichtswissenschaft/Abteilung für Alte Geschichte

Am Hof 1e

53113 Bonn

Tel.: 0228-73-7613

E-Mail: t.shahin@uni-bonn.de 